\title{
Evaluating Postoperative Muscle Strength Using Surface Electromyography in Hip Fracture Patient
}

\author{
Jun-II Yoo ${ }^{1}$, Hayoung Byun ${ }^{2}$, Hyeong Seop Kim², Yun Jeong Jang ${ }^{2}$, Chang Han Lee ${ }^{2}$ \\ 'Department of Orthopaedic Surgery, Gyeongsang National University Hospital, Gyeongsang National University School of Medicine, Jinju; \\ ${ }^{2}$ Department of Rehabilitation Medicine, Gyeongsang National University Hospital, Gyeongsang National University School of Medicine, \\ Jinju, Korea
}

\author{
Corresponding author \\ Chang Han Lee \\ Department of Rehabilitation Medicine, \\ Gyeongsang National University Hospital, \\ Gyeongsang National University School of \\ Medicine, 79 Gangnam-ro, Jinju 52727, Korea \\ Tel: +82-55-750-9639 \\ Fax: +82-55-750-8255 \\ E-mail: ychkhk1407@gmail.com
}

Received: March 7, 2020

Revised: March 20, 2020

Accepted: March 28, 2020
Copyright $\odot 2020$ The Korean Society for Bone and Mineral Research

This is an Open Access article distributed under the terms of the Creative Commons Attribution Non-Commercial License (https://creativecommons.org/licenses/by-nc/4.0/) which permits unrestricted non-commercial use, distribution, and reproduction in any medium, provided the original work is properly cited.

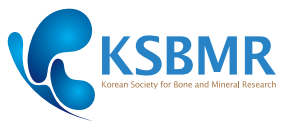

Background: To compare the muscle strength of patients with a hip fracture according to the presence of sarcopenia after surgery and the correlation of measured values between a Biodex and surface electromyography (sEMG) in postoperative measurement of muscle strength. Methods: Seventy-one patients who underwent hip fracture surgery were included in this study. Muscle mass was measured using dual energy X-ray absorptiometry and the grip strength was evaluated using a dynamometer. The diagnosis of sarcopenia followed the Asian Working Group for Sarcopenia criteria. We evaluated the Biodex to assess muscle strength according to the presence of sarcopenia and at the same time measured the sEMG to evaluate the correlation of muscle strength between Biodex and sEMG. Results: We assigned 34 patients with sarcopenia and 37 without sarcopenia to 2 groups. In the comparison of muscle strength using Biodex and SEMG between the 2 groups, it was confirmed that muscle strength of sarcopenia group was decreased compared with that of the non-sarcopenia group, although there was no statistical significance between the groups. However, Biodex and sEMG showed very close correlation with muscle strength in all variables. Conclusions: We suggest that using sEMG for the evaluation of muscle strength after hip fracture surgery may be an excellent tool alternative to isokinetic testing machines such as the Biodex.

Key Words: Electromyography · Hip fracture · Muscle strength · Sarcopenia

\section{INTRODUCTION}

Hip fractures are common in elderly patients. The mortality rate of hip fracture patients is known to be $1.6 \%$ during the hospital stay and $13.5 \%$ at 6 months after discharge. Of these, $12.8 \%$ of patients are known to require full help from others while walking.[1] After hip fracture surgery, patients suffer from disturbances in daily living and walking.[2] Risk factors for death after hip surgery include being elderly, men, comorbidities, cognitive decline, and thromboembolism.[3] The most important factor in restoring the function of hip fracture patients is the ability to walk. However, only $50 \%$ to $65 \%$ restores their previous function, and $10 \%$ to $15 \%$ can only walk indoors.[4] There are reports that early walking helps with functional prognosis, suggesting that early rehabilitation is necessary for functional recovery.[5]

Recently, the concept of sarcopenia can be found not only in studies for geriatric 
diseases but also in various research or clinical fields. Sarcopenia is a geriatric syndrome defined as a gradual decline in muscle function caused by a decrease in skeletal muscle mass.[6] There are many factors related to functional recovery in patients with hip fractures, such as depression, cognitive decline, and delirium. Among them, the presence of sarcopenia was reported to have the most effect on functional recovery after hip fracture.[7]

In patients after hip fracture with sarcopenia, objective examinations are certainly required to determine whether their functions after hip fracture are restored as well as new therapeutic approaches to functional recovery. Many studies have been published in this line. $[1,2,4,5,7]$ However, no research for hip fracture with sarcopenia has been conducted to date. Currently, there are no inspection tools other than measuring grip strength. Pham et al. [8] showed that quadriceps muscle strength declined with advancing age in both women and men, and this reduction was associated with an increased risk of fracture in both sexes. A few studies reported that there was test-retest reliability of Biodex for measuring quadriceps muscle strength for various ages and diseases.[9-12] However, it can be difficult to use the Biodex in patients with hip fracture because of their pain. Hence a convenient approach is necessary for measuring muscle strength of hip fractures with sarcopenia. The use of surface electromyography (sEMG) as a tool to estimate dynamic and isometric muscle forces has been acknowledged. It has been used in both research and clinical applications for non-invasive neuromuscular assessment in several different fields, such as sport science, neurophysiology, and rehabilitation.[13-15]

Therefore, the purpose of this study was to compare the muscle strength of patients with hip fracture according to the presence of sarcopenia after surgery and correlate the measured values between Biodex and SEMG in postoperative measurement of muscle strength. We hypothesized that patients with sarcopenia might show decreased muscle strength measured by Biodex, which would correlate with sEMG.

\section{METHODS}

\section{Participants}

From September 2017 to January 2019, subjects were 65 to 90 years of age or older who underwent surgery for fe- mur neck, intertrochanteric, and subtrochanteric fracture in our institution. The hip surgery methods included bipolar hemiarthroplasty, total hip arthroplasty, or open reduction and internal fixation. The exclusion criteria were as follows: (1) hip surgery for infection, arthritis, implant loosening, or avascular necrosis; (2) femoral shaft fracture, acetabular fracture, isolated fracture of the greater or lesser trochanter, or periprosthetic fracture; (3) pathologic or combined multiple fracture; (4) revision surgery; and (5) severe cognitive dysfunction (one step obey command was not possible). Patients who refused to participate in this study were also excluded.

This study was approved by the Institutional Review Board of our Hospital. All participants provided written informed consent to participate in the study. The clinical trial number of registration is KCT0003843

\section{Measurement for sarcopenia}

The presence or absence of sarcopenia was defined in accordance with the recommendations of the Asian Working Group for Sarcopenia (AWGS).[16] AWGS has agreed with previous reports suggesting that sarcopenia should be described as low muscle mass combined with low muscle strength and/or low physical performance (usual gait speed). In this study, the presence of sarcopenia was defined in accordance with decreased handgrip strength and low muscle mass in a whole body composition test by dual energy X-ray absorptiometry (Hologic Inc., Bedford, MA, USA). It was impossible to measure the exact gait speed, because patients bear limited weight after hip surgery to protect the joint components and provide an optimal healing environment.[17] Bone mineral content, fat mass, and lean soft tissue mass were measured separately for each part of the body, including the arms and legs. We corrected appendicular lean mass (ALM) by substituting lean mass $(L M)$ in the unfractured leg for the LM in the fractured leg, as previously described: corrected $\mathrm{ALM}=(\mathrm{LM}$ in unfractured leg $\times 2)+L M$ in arms.[18,19] The correction was performed to avoid the confounding role of both metal implants (prostheses, plates, screws, and nails) and postoperative edema at the fractured femur. When we analyzed the muscle mass, the suggested cut-off values ALMs/ height ${ }^{2}$ $\left(\mathrm{kg} / \mathrm{m}^{2}\right)$ are $7.0 \mathrm{~kg} / \mathrm{m}^{2}$ for men and $5.4 \mathrm{~kg} / \mathrm{m}^{2}$ for women. [19]

Muscle strength was assessed by handgrip strength. The 
participant held a digital hand dynamometer (Digital grip strength dynamometer, T.K.K 5401; Takei Scientific Instruments Co., Ltd., Tokyo, Japan) in the sitting position. The elbow was flexed at 90 degrees with the shoulder attached to the torso, with the wrist maintaining a neutral posture $(0$ degrees), and the maximal grip strength was measured. Sarcopenia was defined according to the AWGS criteria for low muscle strength (handgrip strength below $18 \mathrm{~kg}$ in women and below $26 \mathrm{~kg}$ in men).[16]

\section{Isokinetic knee strength examination}

The Knee strength was measured by placing the subject in a knee joint measuring chair of an isokinetic muscle strength measuring instrument (Biodex Medical System, Shirley, NY, USA) (Fig. 1). The examiner placed a knee stabilizer pad on the subject's ankle and fixed the chest, abdomen, and femur with a fixed band so that no external force was applied to the pelvis and femoral muscle. The measurement of knee strength was performed in the non-affected side (non-operated side). The peak torques among the isokinetic parameters at angular velocities of $60 \% \mathrm{~s}$ ( 5 times) and $180 \% \mathrm{~s}$ (10 times) were measured and analyzed. To allow adaptation prior to the examination, subjects were allowed to exercise 3 times prior to testing. A resting time of 2 min was allowed between tests of each angular velocity. During the tests, patients were encouraged to perform with maximum effort by the examiner.

\section{Assessment of surface EMG}

The sEMG was performed by attaching electrodes to the knee flexors and extensors (biceps femoris and rectus fem-

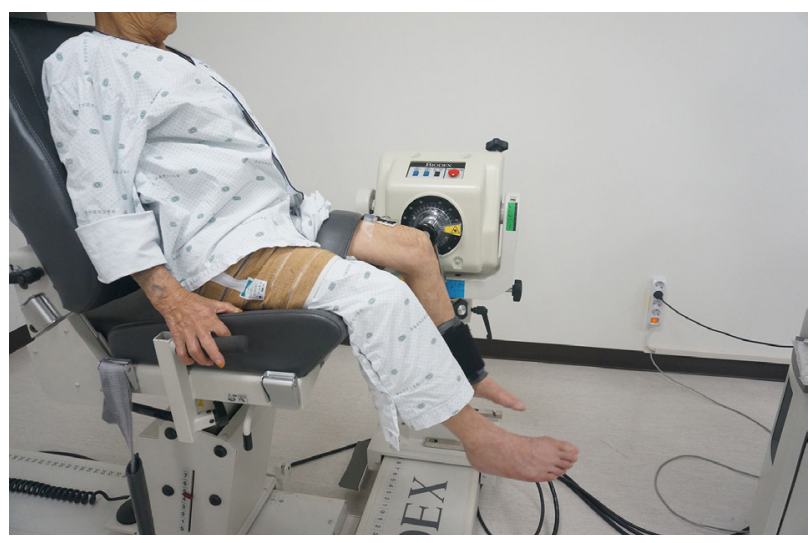

Fig. 1. Muscle strength was tested using the Biodex and surface electromyography. oris) when Biodex was used. B-mode ultrasonography (Philips Medical Systems, Bothell, WA, USA) with an L12-5 linear transducer was used to identify specific electrodes placement location. The validity of the protocol is reported elsewhere.[20] A wireless sEMG system (Noraxon Telemyo DTS system; Noraxon USA Inc., Scottsdale, AZ, USA) were used to perform 3-dimensional motion analysis and measure the muscle activation level. Gel-type, 20-mm diameter, Ag/ $\mathrm{AgCl}$ alloy dual electrodes (Noraxon USA Inc.) were attached to biceps femoris and rectus femoris muscles of the nonaffected side (non-operated side) after cleaning the sites with alcohol to reduce skin impedance. The SEMG was amplified 500 times, sampled at $1.5 \mathrm{kHz}$ and digitized using a 16-bit analogue to digital converter. Signals were filtered at a bandwidth of 10 to $500 \mathrm{~Hz}$ to eliminate noise recorded during the data collection process, and root-mean-square window of $50 \mathrm{~ms}$ was used for signal smoothing. Mean amplitude of all period ( $\mathrm{UV}$ ) was used for analysis.

\section{Statistical analysis}

SPSS Statistics version 23 (SPSS Inc., Chicago, IL, USA) for Windows was used for statistical analysis. Continuous variables were expressed as means and standard deviations with percentages. To compare means and proportions between groups, a Student's $t$-test and $\chi^{2}$ test were performed. Pearson correlation analysis was done to evaluate the relationship of measured values between Biodex and sEMG, and the significance level was set at $P<0.05$. A post hoc power analysis was performed with a correlation analysis to determine the power of our statistical findings. With the total sample size at a minimum of 59 patients and the a significance level and effect size set at 0.05 and 0.4 , respectively, the study's power was determined to be no less than 0.952 for any significant difference found.

\section{RESULTS}

\section{General characteristics of subjects}

A total of 115 patients underwent hip fracture surgery were screened for the trial, of whom 71 were eligible. Of these patients, 34 were assigned to sarcopenia; 37 were assigned to non-sarcopenia group. Figure 2 shows the flow of participants' enrollment in the study. The mean age of the subjects was $71.4 \pm 11.9$ years for non-sarcopenia and $73.5 \pm 11.3$ years for sarcopenia. Females made up $66.6 \%$ 


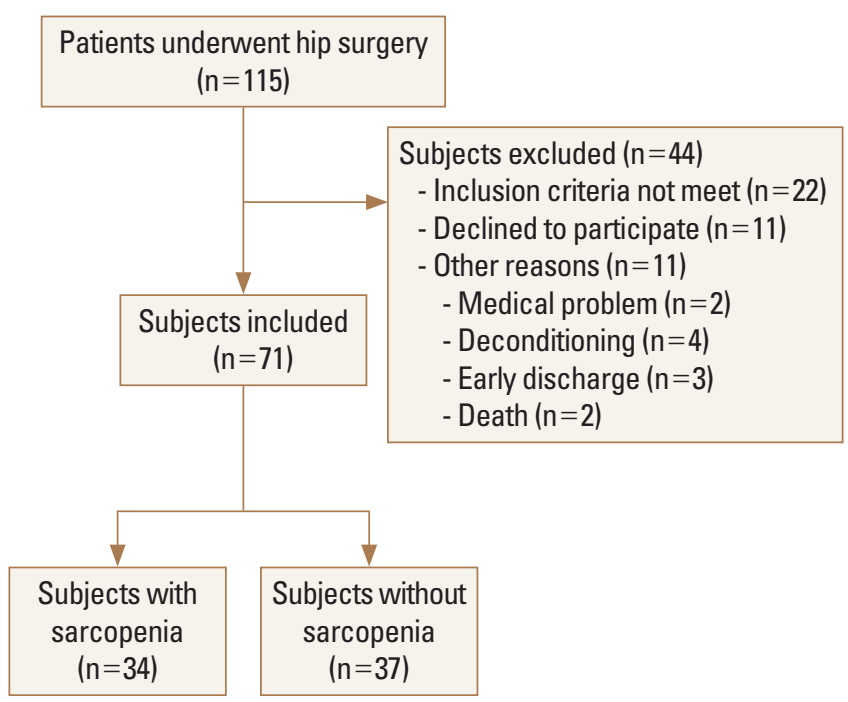

Fig. 2. Flow chart showing subject numbers at each step of the study.

Table 1. Demographic findings

\begin{tabular}{|c|c|c|c|}
\hline Variables & $\begin{array}{c}\text { Non-sarcope- } \\
\text { nia }(n=37)\end{array}$ & $\begin{array}{l}\text { Sarcopenia } \\
\quad(n=34)\end{array}$ & $P$-value \\
\hline Age (yr) & $71.4 \pm 11.9$ & $73.5 \pm 11.3$ & 0.545 \\
\hline Sex & & & 0.123 \\
\hline Women & $21(56.8)$ & $27(79.4)$ & \\
\hline Men & $16(43.2)$ & 7 (20.6) & \\
\hline Hip fracture type & & & 0.068 \\
\hline Femoral neck & $18(48.6)$ & $11(32.4)$ & \\
\hline Intertrochanteric & $13(35.1)$ & $22(64.7)$ & \\
\hline Subtrochanteric & $6(16.2)$ & $1(2.9)$ & \\
\hline Operation & & & 0.952 \\
\hline THA & $12(32.4)$ & $11(32.4)$ & \\
\hline $\mathrm{BHA}$ & $14(37.8)$ & 12 (35.3) & \\
\hline Internal fixation & $11(29.7)$ & $11(32.4)$ & \\
\hline ALM/height ${ }^{2}\left(\mathrm{~kg} / \mathrm{m}^{2}\right)$ & $6.22 \pm 1.12$ & $5.08 \pm 0.52$ & 0.019 \\
\hline Female & $5.62 \pm 0.49$ & $5.05 \pm 0.55$ & 0.548 \\
\hline Male & $7.06 \pm 1.18$ & $5.11 \pm 0.45$ & 0.106 \\
\hline PTH (ng/dL) & $50.5 \pm 21.0$ & $63.3 \pm 50.4$ & 0.285 \\
\hline Vitamin D (ng/dL) & $15.5 \pm 8.7$ & $15.3 \pm 10.3$ & 0.949 \\
\hline Hip T score & $-1.3 \pm 1.1$ & $-1.8 \pm 1.2$ & 0.159 \\
\hline Spine T score & $-0.9 \pm 1.6$ & $-1.6 \pm 1.7$ & 0.216 \\
\hline \multicolumn{2}{|l|}{ Pre-fracture ambulation capacity } & & 1.000 \\
\hline Independent ambulation & $36(97.3)$ & $33(97.1)$ & \\
\hline Ambulation with assistance & $1(2.7)$ & $1(2.9)$ & \\
\hline
\end{tabular}

THA, total hip arthroplasty; BHA, bipolar hemiarthroplasty; ALM, appendicular lean mass; PTH, parathyroid hormone.

of the population (Table 1). We assigned 34 patients with sarcopenia and 37 without sarcopenia to 2 groups. There
Table 2. Muscle strength and masticatory power by presence of sarcopenia

\begin{tabular}{lccc}
\hline Variables & $\begin{array}{c}\text { Non-sarcopenia } \\
(\mathrm{n}=37)\end{array}$ & $\begin{array}{c}\text { Sarcopenia } \\
(\mathrm{n}=34)\end{array}$ & $P$-value \\
\hline B_BicepsK60 & $14.1 \pm 12.0$ & $11.0 \pm 14.7$ & 0.427 \\
B_RectusK60 & $36.7 \pm 29.3$ & $30.4 \pm 33.7$ & 0.497 \\
B_BicepsK180 & $10.5 \pm 10.9$ & $7.4 \pm 8.3$ & 0.276 \\
B_RectusK180 & $24.6 \pm 18.4$ & $20.3 \pm 18.9$ & 0.436 \\
S_BicepsK60 (uV) & $48.0 \pm 38.8$ & $38.1 \pm 47.6$ & 0.431 \\
S_RectusK60 (uV) & $88.0 \pm 115.0$ & $86.9 \pm 77.9$ & 0.969 \\
S_BicepsK180 (uV) & $65.0 \pm 122.1$ & $54.8 \pm 48.2$ & 0.720 \\
S_RectusK180 (uV) & $117.6 \pm 151.3$ & $90.6 \pm 144.4$ & 0.534
\end{tabular}

B, Biodex electromyography; S, surface electromyography; $K$, knee.

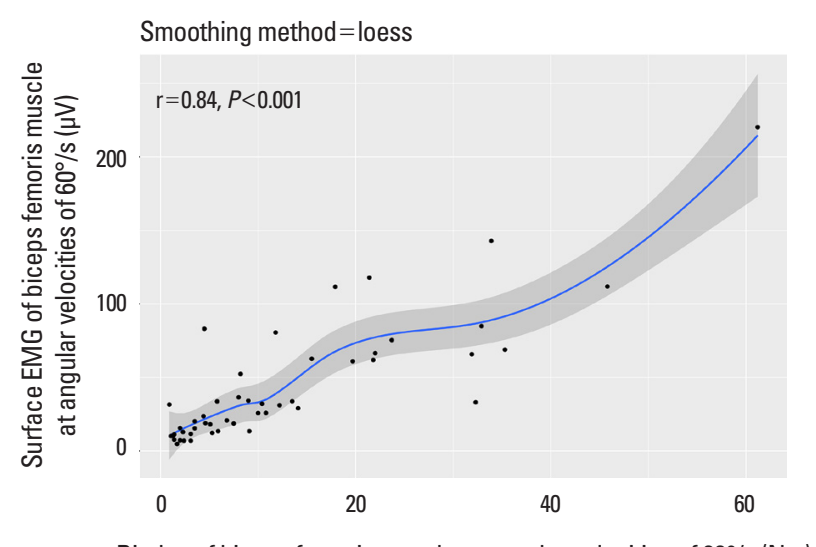

Biodex of biceps femoris muscle at angular velocities of $60 \% \mathrm{~s}(\mathrm{Nm})$

Fig. 3. Correlation coefficient between mean amplitude of surface electromyography (sEMG) and the strength (peak torque) of Biodex in the flexor muscle (biceps femoris) at angular velocities of $60^{\circ} / \mathrm{s}$.

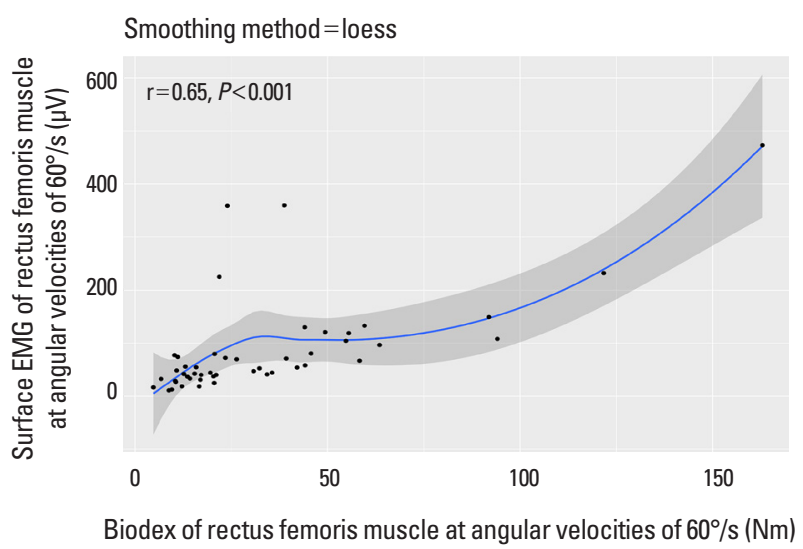

Fig. 4. Correlation coefficient between mean amplitude of surface electromyography (sEMG) and the strength (peak torque) of Biodex in the extensor muscle (rectus femoris) at angular velocities of $60 \%$ s.

was no significant difference between 2 groups in preoperative walking ability (Table 1 ). 


\section{JBM}

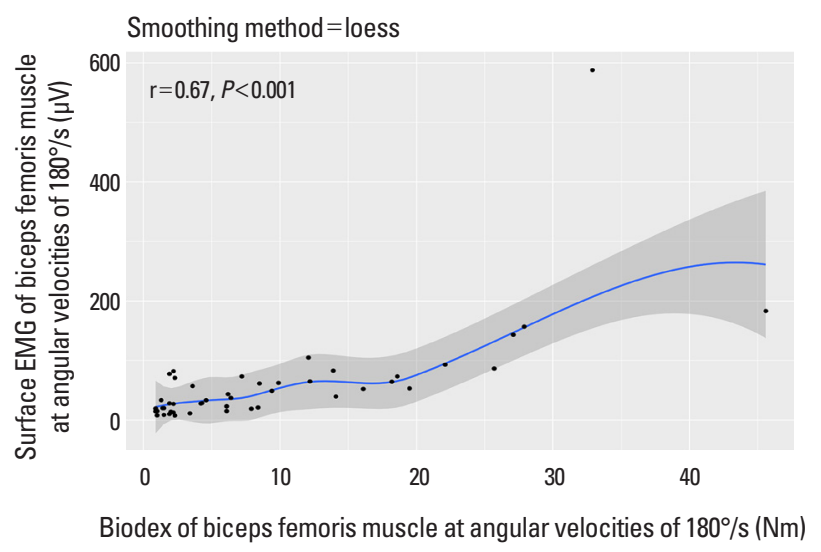

Fig. 5. Correlation coefficient between mean amplitude of surface electromyography (sEMG) and the strength (peak torque) of Biodex in the flexor muscle (biceps femoris) at angular velocities of $180^{\circ} / \mathrm{s}$.

\section{Muscle strength in according to the sarcopenia}

Muscle strength of all participants was measured within 3 to 7 days after hip fracture surgery. In the comparison of muscle strength of knee flexion and extension using Biodex and sEMG between the 2 groups, we confirmed that the muscular strength of sarcopenia group decreased compared with that of non-sarcopenia group, although there was no statistically significant between the 2 groups (Table 2).

\section{Correlation between Surface EMG and Biodex}

There was a correlation between mean amplitude of sEMG and the strength (peak torque) of flexor muscle (biceps femoris) or extensor muscle (rectus femoris) at angular velocities of $60 \%$ on non-affected knee (for flexor and extensor muscles of non-affected side knee at $60 \% \mathrm{~s} r=0.84$, $P<0.001$ and $r=0.65, P<0.001$, respectively) (Fig. 3, 4). At angular velocities of $180 \%$, the mean amplitude of sEMG and the strength of flexor muscle (biceps femoris) on nonaffected knee showed a correlation coefficient of $0.67(P<$ 0.001) (Fig. 5). In addition, There was a mild correlation between mean amplitude of sEMG and the strength of extensor muscle (rectus femoris) at angular velocities of $180 \%$ on non-affected knee $(r=0.23, P=0.12)$, although there was no statistical significance (Fig. 6).

\section{DISCUSSION}

The principle findings of this study was that muscle stren-

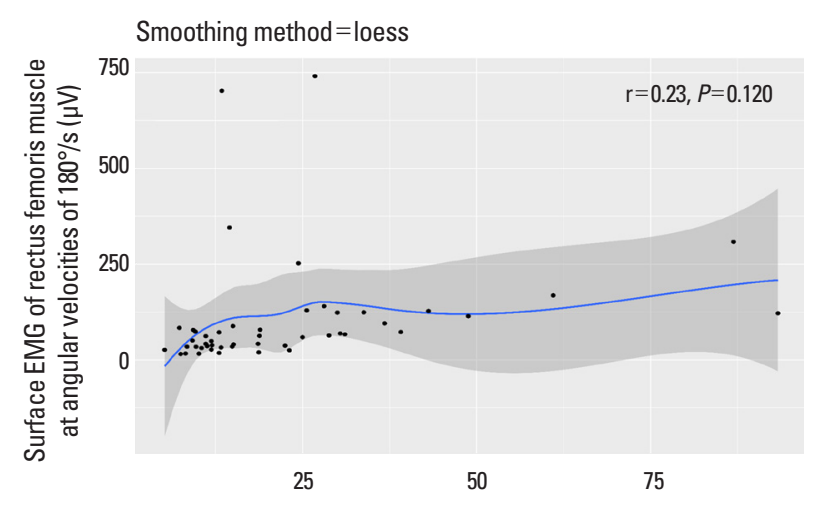

Biodex of rectus femoris muscle at angular velocities of $180 \%$ s (Nm)

Fig. 6. Correlation coefficient between mean amplitude of surface electromyography (sEMG) and the strength (peak torque) of Biodex in the extensor muscle (rectus femoris) at angular velocities of $180^{\circ} / \mathrm{s}$.

gth of rectus femoris and biceps femoris measured by sEMG was statistically associated with one measured by Biodex in patients after hip fracture surgery. When we compared the muscle strength in sarcopenia group and one in the non-sarcopenia group, muscle strength was relatively low in sarcopenia group, although there was no statistical significance. When we analyzed the muscle mass of non-sarcopenia group, ALMs $/ \mathrm{height}^{2}\left(\mathrm{~kg} / \mathrm{m}^{2}\right)$ of females and males was $5.62 \pm 0.49$ and $7.06 \pm 1.18$ respectively. These values were close to the cut-off values of sarcopenia. This result may be attributed to the absence of a significant difference. Especially mean amplitude differences of sEMG were smaller than the muscle strength measured by Biodex between sarcopenia and non-sarcopenia groups, probably because of the problem of positioning sEMG electrodes. Rainoldi and Melchiorri identified optimal electrode sites for muscles in the lower extremity by providing a standard landmarking technique for the localization of the innervation zone of each muscle, so that SEMG electrodes could be properly positioned between the innervation and a tendon.[21] In this study, we didn't take these points into consideration.

Sarcopenia and hip fractures are highly related and increasing muscular strength enhancement is important for post-operative function in patients with hip fracture. The loss of muscle mass and strength puts elderly persons at a high risk for falls and fractures.[22] It has been estimated that approximately $30 \%$ of elderly people dwelling in a community aged 65 and older fall at least once a year, and half of them fall recurrently.[23,24] The most important 
risk factor for falls in elderly individuals is low muscle strength, particularly of the lower extremities.

The amount of decrease in muscular strength may significantly affect postoperative functioning. The quadriceps muscle especially absorbs the impacts on the joints and stabilizes the loaded leg. Therefore, the weakness of the quadriceps muscles caused insufficient impact on the lower extremities, which caused the excessive stress on the lower extremities as the cause of hip fracture.[25] Also, it is known that knee effusion occurs frequently after hip fracture.[26,27] Segal and Glass [28] concluded that greater quadriceps muscle strength protected against incident symptomatic knee osteoarthritis. Coordinated muscle contractions with a healthy balance between agonist and antagonist muscles around the knee during the low-load walking could improve the functional capacity,[29] which showed the importance of the hamstring muscles as well as the quadriceps muscles. Although there was no statistical significance in this study, the muscle strength of the rectus and biceps muscles tended to be low in the sarcopenia group.

Biodex is the most widely used method of measuring the muscle strength of limbs. Since the introduction of isokinetic dynamometry with the Biodex system in 1960,[30] this has become an integral part of testing and rehabilitation of injuries to the upper and lower extremities.[31,32] The reliability of the Biodex system has been assessed for the measurement of concentric and eccentric loading in lower extremities.[33,34] To assess such changes in muscle performance, accurate, reliable, and applicable methods to evaluate muscle performance should be applied. However, muscle strength assessment using Biodex can be difficult for all patients because of pain after surgery. In this study, all participants are especially patients within 3 to 7 days after surgery. This is why we performed the measurement of knee strength in the non-affected side (non-operated side). A simple protocol is especially needed for older patients when assessing their strength after surgery.

Electromyography refers to the collective electric signal from muscles, which is controlled by the nervous system and produced during muscle contraction. The signal represents the anatomical and physiological properties of muscles. In fact, an electromyography signal is the electrical activity of a muscle's motor units and is most useful as electrophysiological signals in medical fields.[35] The electrical activity of motor units can be recorded by non-invasive (sEMG) electrodes and invasive (intramuscular electromyography) electrodes, respectively. Currently, surface-detected signals are preferably used to obtain information about the time or intensity of superficial muscle activation. [36] When SEMG is used, careful interpretation is required with regard to the proper placement of the electrode, presence of subcutaneous adipose tissue, crosstalk of unintended muscle activation, and signal cancellation of asynchronous action potential firing. In this study, Biodex and sEMG values were highly correlated. In particular, there was a high correlation with the measured muscle strength of the lower extremity when the patient performed the extension and flexion of the knee joint.

There are several limitations to this study. First, sample size was relatively small. This is attributed to our stringent inclusion and exclusion criteria. Second, for Biodex, this was a closed kinetic chain study, and thus, the results obtained may differ from those of real knee, which have closed and open components. Third, mean amplitude of all period was used for analysis in the measurements of sEMG. Because mean area of all period also can be measured simultaneously, additional studies will be needed to establish which measurement is best. Fourth, the types of surgery included in the study were different from each other. In addition, we measured and correlated the knee strength, while other muscles such as hip abductor might also have important role for gait. This was because hip abduction was not safe for some patients after surgery, and Biodex had limitations on measuring various hip muscle strengths. Future study with more diverse muscle strength measurements might be useful. Finally, for sEMG, optimal electrode sites for muscles in the lower extremity were not considered and duly weighed. However, we tried to minimize the invalidity using the ultrasound. Additional studies providing a standard landmarking technique for the optimal electrode sites will be needed.

Although was no statistically significant difference in muscle strength with or without sarcopenia, the values of Biodex and SEMG were highly correlated. This is the first study which used the Biodex and SEMG to measure and correlate the muscle strength in patients with and without sarcopenia after hip fracture. Therefore, we suggest that the use of sEMG for the evaluation of muscle strength after hip fracture surgery may be an excellent tool alternative to 
isokinetic testing machines such as the Biodex, with less pain and more convenience.

\section{DECLARATIONS}

\section{Ethics approval and consent to participate}

All procedures performed in studies involving human participants were in accordance with the ethical standards of the institutional and/or national research committee and with the 1964 Helsinki declaration and its later amendments or comparable ethical standards. Informed consent was obtained from all individual participants included in the study.

\section{Conflict of interest}

No potential conflict of interest relevant to this article was reported.

\section{ORCID}

Jun-II Yoo https://orcid.org/0000-0002-3575-4123

Hayoung Byun https://orcid.org/0000-0003-1426-6582

Hyeong Seop Kim https://orcid.org/0000-0001-5022-6030

Yun Jeong Jang https://orcid.org/0000-0002-6406-5351

Chang Han Lee https://orcid.org/0000-0001-8351-5226

\section{REFERENCES}

1. Lyons AR. Clinical outcomes and treatment of hip fractures. Am J Med 1997;103:51S-63S.

2. Magaziner J, Fredman L, Hawkes W, et al. Changes in functional status attributable to hip fracture: a comparison of hip fracture patients to community-dwelling aged. Am J Epidemiol 2003;157:1023-31.

3. Sellier E, Labarere J, Sevestre MA, et al. Risk factors for deep vein thrombosis in older patients: a multicenter study with systematic compression ultrasonography in postacute care facilities in France. J Am Geriatr Soc 2008;56:224-30.

4. Hannan EL, Magaziner J, Wang JJ, et al. Mortality and locomotion 6 months after hospitalization for hip fracture: risk factors and risk-adjusted hospital outcomes. JAMA 2001;285:2736-42.

5. Siu AL, Penrod JD, Boockvar KS, et al. Early ambulation after hip fracture: effects on function and mortality. Arch Intern Med 2006;166:766-71.

6. Clynes MA, Edwards MH, Buehring B, et al. Definitions of sarcopenia: associations with previous falls and fracture in a population sample. Calcif Tissue Int 2015;97:445-52.

7. Landi F, Cesari M, Calvani R, et al. The "Sarcopenia and Physical fRailty IN older people: multi-componenT Treatment strategies" (SPRINTT) randomized controlled trial: design and methods. Aging Clin Exp Res 2017;29:89-100.

8. Pham HM, Nguyen ND, Center JR, et al. Contribution of quadriceps weakness to fragility fracture: a prospective study. J Bone Miner Res 2016;31:208-14.

9. Roberts D, Ageberg E, Andersson G, et al. Clinical measurements of proprioception, muscle strength and laxity in relation to function in the ACL-injured knee. Knee Surg Sports Traumatol Arthrosc 2007;15:9-16.

10. Tsiros MD, Grimshaw PN, Schield AJ, et al. Test-retest reliability of the biodex system 4 Isokinetic Dynamometer for knee strength assessment in paediatric populations. J Allied Health 2011;40:115-9.

11. Feiring DC, Ellenbecker TS, Derscheid GL. Test-retest reliability of the biodex isokinetic dynamometer. J Orthop Sports Phys Ther 1990;11:298-300.

12. Flansbjer UB, Downham D, Lexell J. Knee muscle strength, gait performance, and perceived participation after stroke. Arch Phys Med Rehabil 2006;87:974-80.

13. Merletti R, Rainoldi A, Farina D. Surface electromyography for noninvasive characterization of muscle. Exerc Sport Sci Rev 2001;29:20-5.

14. De Luca CJ. The use of surface electromyography in biomechanics. Journal of Applied Biomechanics 1997;13:13563.

15. De Luca CJ. Use of the surface EMG signal for performance evaluation of back muscles. Muscle Nerve 1993;16:210-6.

16. Chen LK, Liu LK, Woo J, et al. Sarcopenia in Asia: consensus report of the Asian Working Group for Sarcopenia. J Am Med Dir Assoc 2014;15:95-101.

17. Rath E, Sharfman ZT, Paret M, et al. Hip arthroscopy protocol: expert opinions on post-operative weight bearing and return to sports guidelines. J Hip Preserv Surg 2017;4: 60-6.

18. Di Monaco M, Castiglioni C, Vallero F, et al. Sarcopenia is more prevalent in men than in women after hip fracture: a cross-sectional study of 591 inpatients. Arch Gerontol Geriatr 2012;55:e48-52.

19. Di Monaco M, Vallero F, Di Monaco R, et al. Muscle mass and functional recovery in men with hip fracture. Am J Phys Med Rehabil 2007;86:818-25. 
20. Kellis E, Galanis N, Natsis K, et al. Validity of architectural properties of the hamstring muscles: correlation of ultrasound findings with cadaveric dissection. J Biomech 2009; 42:2549-54.

21. Rainoldi A, Melchiorri G, Caruso I. A method for positioning electrodes during surface EMG recordings in lower limb muscles. J Neurosci Methods 2004;134:37-43.

22. Landi F, Liperoti R, Russo A, et al. Sarcopenia as a risk factor for falls in elderly individuals: results from the ilSIRENTE study. Clin Nutr 2012;31:652-8.

23. Tinetti ME, Speechley M, Ginter SF. Risk factors for falls among elderly persons living in the community. $\mathrm{N}$ Engl J Med 1988;319:1701-7.

24. Graafmans WC, Ooms ME, Hofstee HM, et al. Falls in the elderly: a prospective study of risk factors and risk profiles. Am J Epidemiol 1996;143:1129-36.

25. Øiestad BE, Juhl CB, Eitzen I, et al. Knee extensor muscle weakness is a risk factor for development of knee osteoarthritis. A systematic review and meta-analysis. Osteoarthritis Cartilage 2015;23:171-7.

26. Christodoulou AG, Givissis P, Antonarakos PD, et al. Knee joint effusion following ipsilateral hip surgery. J Orthop Surg (Hong Kong) 2010;18:309-11.

27. Shahid MS, Murphy D, O'Donnell T, et al. A prospective study for evaluation of knee effusion after hip surgery. Ir Med J 2002;95:140-1.

28. Segal NA, Glass NA. Is quadriceps muscle weakness a risk factor for incident or progressive knee osteoarthritis? Phys
Sportsmed 2011;39:44-50.

29. Peeler J, Christian M, Cooper J, et al. Managing knee osteoarthritis: the effects of body weight supported physical activity on joint pain, function, and thigh muscle strength. Clin J Sport Med 2015;25:518-23.

30. Hislop HJ, Perrine JJ. The isokinetic concept of exercise. Phys Ther 1967;47:114-7.

31. Wyatt MP, Edwards AM. Comparison of quadriceps and hamstring torque values during isokinetic exercise. J Orthop Sports Phys Ther 1981;3:48-56.

32. Humer M, Kosters A, Müller E. Dynamic and static maximum strength in closed kinetic chain movements. Trunkflexion/-extension and -rotation. Sportverletz Sportschaden 2011;25:13-21.

33. Tredinnick TJ, Duncan PW. Reliability of measurements of concentric and eccentric isokinetic loading. Phys Ther 1988; 68:656-9.

34. Frisiello S, Gazaille A, O'Halloran J, et al. Test-retest reliability of eccentric peak torque values for shoulder medial and lateral rotation using the Biodex isokinetic dynamometer. J Orthop Sports Phys Ther 1994;19:341-4.

35. Farina D, Negro F. Accessing the neural drive to muscle and translation to neurorehabilitation technologies. IEEE Rev Biomed Eng 2012;5:3-14.

36. Chowdhury RH, Reaz MB, Ali MA, et al. Surface electromyography signal processing and classification techniques. Sensors (Basel) 2013;13:12431-66. 